\title{
HEMP Protection of Electronic Equipment Located in Control Cabinets
}

\author{
Vladimir Gurevich, Ph.D.* \\ Central Electrical Laboratory Israel Electric Corp., Haifa, Israel \\ *Corresponding Author: Vladimir Gurevich, Central Electrical Laboratory Israel Electric Corp., Haifa, \\ Israel
}

\begin{abstract}
This article discusses the effect of location and design of varistor-based protection modules regarding the efficiency of electronic equipment protection from high-altitude electromagnetic pulse at nuclear explosion (HEMP) and presents specific technical solutions which ensure maximum efficiency of protection.
\end{abstract}

Keywords: Electromagnetic pulse, HEMP, protection of electronic equipment, control cabinets, varistor modules

\section{INTRODUCTION}

It is commonly accepted in industrial and power sectors that electronic devices of automatic measurement and control, as well as digital protection relays (DPR), should be placed in metal cabinets. In order to improve HEMP-resistance of electronic equipment placed in cabinets, the design of the cabinets should be very specific [1]. Firstly, it is preferable that the doors of the cabinets should be made from metal, rather than glass that they are usually made of (Fig1).

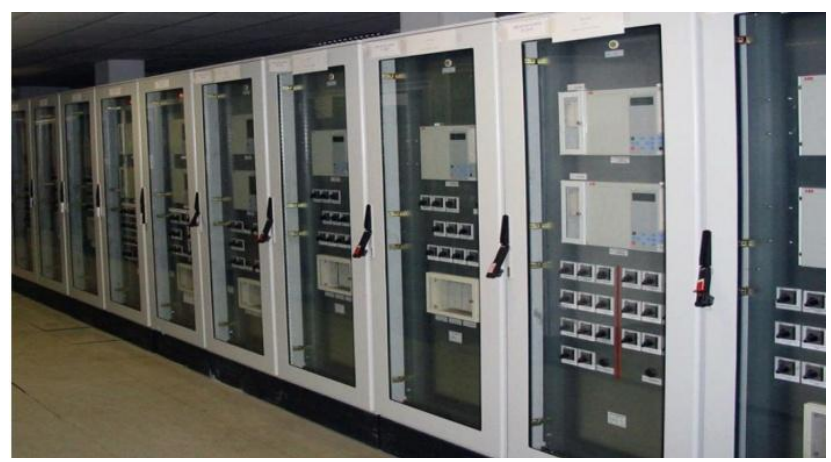

Figure1. Common placement of electronic equipment, in particular, digital protection relay (DPR) in glassdoor cabinets.

Secondly, the doors should be lined with conductive rubber sealers along the perimeter. Thirdly, air ventilation inlets in these cabinets should be covered with honeycomb structure blocks (waveguidesbelow-cutoff).

These measures are intended to reduce electromagnetic emissions entering a cabinet and to prevent failures of electronic equipment. However, many of the multi-core cables that penetrate into the cabinet from outside and are connected to input and output terminals of sensitive electronic equipment make the above-mentioned measures insufficient. Apart from protecting electronic equipment from external electromagnetic emission, there is a need for additional protection from pulse voltage of the nano-second range, which upon HEMP impact flows directly to input and output terminals of equipment located in the cabinet via the external cables. As shown in [1], varistors and TVS-diodes connected parallel to input terminals are efficient in protecting electronic equipment from pulse overvoltage entering its input and output terminals upon HEMP impact. There can be more than several dozens of input and output terminals in one cabinet. The number of protection elements should be the same. In this situation, optimum design of modules containing protection elements and 
their location in the cabinet becomes very relevant. It is especially true for retrofit when ensuring protection of existing cabinets rather than newly designed models. These issues are addressed in this article.

\section{SELECTING THE DESIGN OF PROTECTION MODULES}

Varistors and TVS-diodes represent electronic components intended to be installed on printed circuit boards, whereas inputs and outputs of electronic equipment located in the cabinets are intended for connecting mounting wires by screws (Fig2). At the same time, various types of equipment can significantly differ from each other in terms of terminal block design, which doesn't allow the use of all-purpose universal connectors instead of ordinary mounting wires.
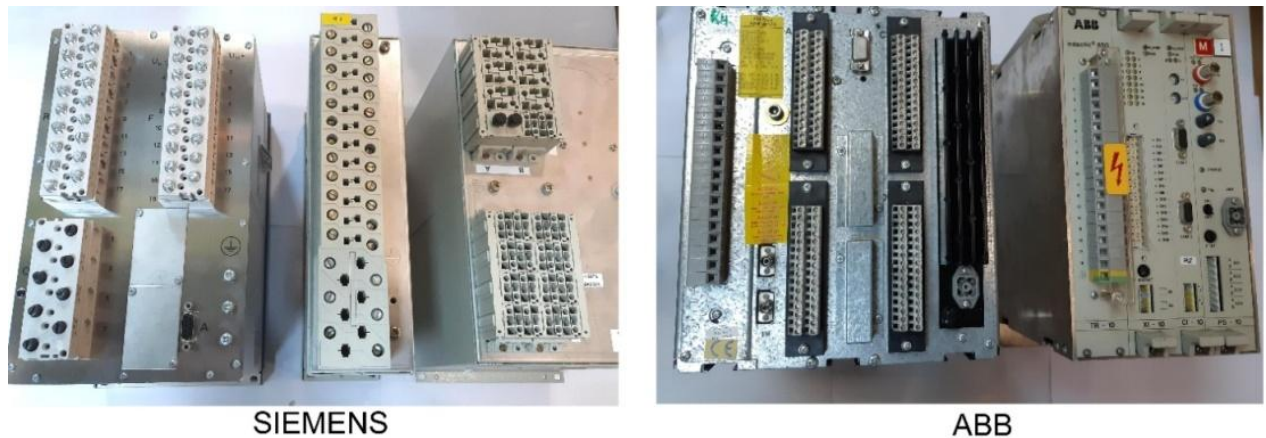

Figure2. Design of terminal assembly (connectors) of digital protection relays (DPR) or various types and from various manufacturers.

Thus, the first issue is the problem of ensuring the connection between protection elements located on printed circuit boards and terminal blocks of electronic equipment. Actually, there are several solutions, e.g. use of a simple in-house manufactured adapter panel utilizing a printed circuit board with protecting elements and terminals (Fig3).

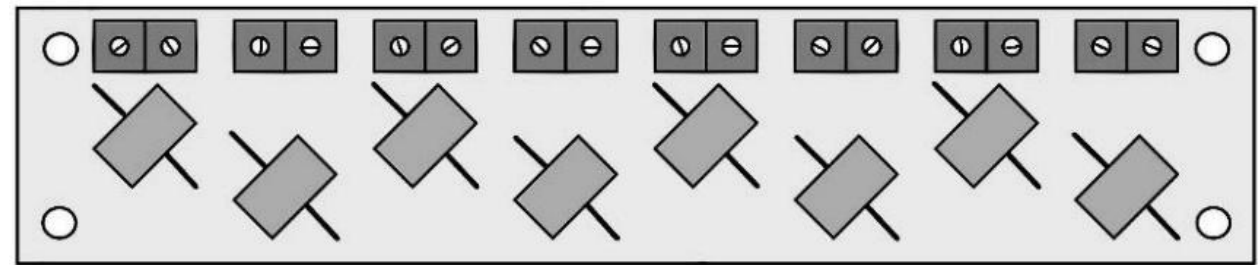

Figure3. Sample of a simple self-made circuit board with protection elements (varistors or TVS-diodes) and standard terminals.

Alternatively, one can use varistor blocks in plastic housing designed for mounting on a standard DIN rail (Fig4). These blocks are convenient for mounting in cabinets and are distributed by various manufacturers, such as: Siemens, Schneider Electric, Weidmuller, Cooper Bussmann, Wago, Phoenix Contact, Citel, Relpol and many others.
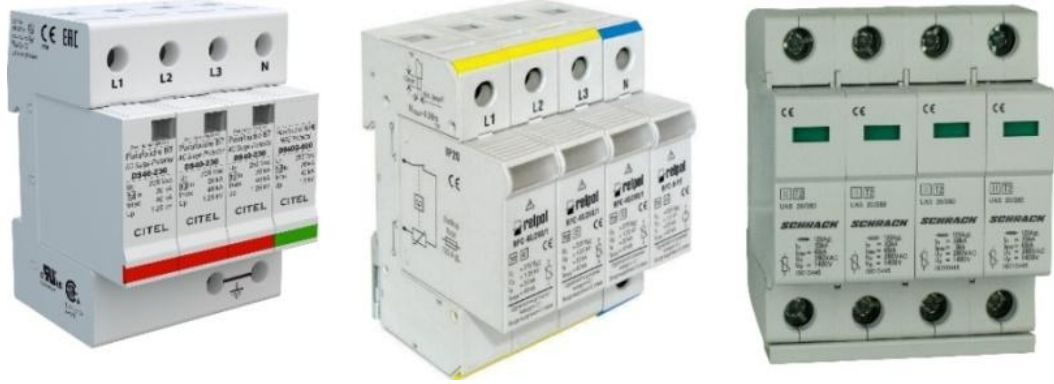

Figure4.Varistor blocks designed for mounting in cabinets on a standard DIN rail manufactured by different companies.

Unfortunately, the dimensions of these blocks for several dozens of varistors will be too large and this makes them practically impossible to be used in existing cabinets with equipment.

One of the solutions would be the use of fabricated modules containing varistors and standard terminals for mounting cables densely mounted on a printed circuit board (Fig5.)

International Journal of Research Studies in Electrical and Electronics Engineering (IJRSEEE) Page $\mid 2$ 


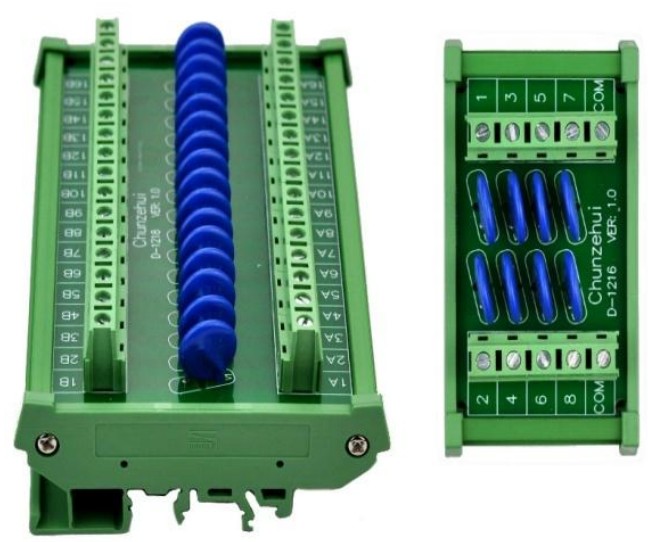

Figure5.Modules with various numbers of varistors manufactured by Chunzehui Company designed for mounting in cabinets on a standard DIN rail.

These varistors will occupy significantly less space compared to varistor blocks shown in Fig. 4,thus they are more preferred for retrofitting ofexisting cabinets with electronic equipment.

\section{Efficiency Of Protection Elements As Affected By Mounting Wires}

What is the best place to mount the modules inside a control cabinet? The answer seems to be obvious: near to the equipment which they need to protect in order to reduce the length of additional mounting wires between the modules and equipment. But what should be the practical shortest distance between these modules and equipment, and what can be the minimum length of additional mounting wires between each varistor and each input (output) of equipment being protected. Unfortunately, it is not so easy to find an empty space in a cabinet full of equipment, even for relatively small modules (Fig5) and to maintain a minimum length of the mounting wires. However, since we deal with a very short current pulse falling within a nano-second range, i.e. corresponding to a high-frequency range, obviously there is a need to address the issue of protection efficiency as affected by the length of mounting wires between the protection elements and equipment being protected.

There are different ways to calculate inductance of individual conductors, and regarding this there are different formulas. However, experience has shown that the results of calculations using different formulas are close and thus any of these methods will be good for our estimates, e.g. the one offered in [2]:

$L_{S W}=2 l\left\{\ln \left[\left(\frac{2 l}{D}\right)\left(1+\sqrt{1+\left(\frac{D}{2 l}\right)^{2}}\right)\right]-\sqrt{1+\left(\frac{D}{2 l}\right)^{2}}+\frac{\mu}{4}+\left(\frac{D}{2 l}\right)\right\}$

Where $L_{S W}$ is the inductance of a single straight conductor with length $l$ and diameter $D$.

Since the larger the diameter, the lower the inductance of a conductor, $4 \mathrm{~mm}^{2}$ was taken as the maximum practically possible cross section of mounting wires. The diameter of a wire was determined as:

$$
D=\sqrt{\frac{4 C S}{\pi}}=\sqrt{\frac{4 \times 4}{3.14}}=2.26 \mathrm{~mm}
$$

Where $\mathrm{D}$ is the diameter of a wire, CS is the cross section.

It is also known that in order to reduce inductance of wires they need to be twisted in pairs (so called "twisted pair") (Fig6) and this can be used in our case. However, this necessitates accounting for mutual inductance between two conductors, in other words, inductance of a loop established by two twisted wires connected in series:

$\mathrm{L}_{\mathrm{PL}}=\frac{\mu \mu_{0}}{\pi} \cosh ^{-1}\left(\frac{\mathrm{S}}{\mathrm{D}}\right) \mathrm{l}$

Where: $L_{P L^{-}}$- inductance of a loop established by a twisted pair of conductors;

$S$ - Distance between conducting cores;

$D$ - Diameter of a conducting core;

International Journal of Research Studies in Electrical and Electronics Engineering (IJRSEEE) Page | 3 
$l$ - Length of a conductor; $\cosh (x)=\frac{e^{x}+e^{-x}}{2}-$ Hyperbolic cosine.

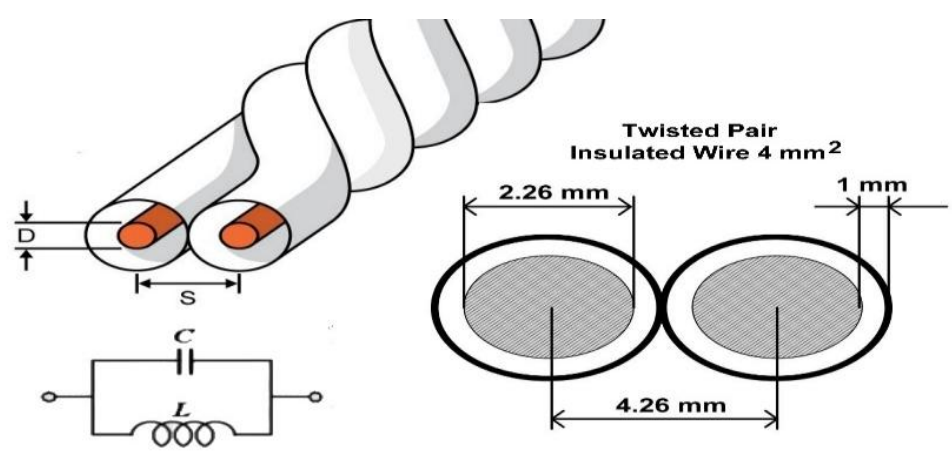

Figure6. Estimation model for a twisted pair of conductors.

It is also obvious that the capacitance between conductors creates a significant effect which should also be accounted for. The results of calculations under the above-mentioned formulas are shown in Table1.

Table1. Inductance and capacitance of conductors connecting a varistor with equipment being protected

\begin{tabular}{|c|c|c|c|}
\hline $\begin{array}{c}\text { Length of wire, } \\
\mathrm{m}\end{array}$ & $\begin{array}{c}\text { Inductance of two individual } \\
\text { wires, } \\
\mathrm{nH}\end{array}$ & $\begin{array}{c}\text { Full inductance of the } \\
\text { twisted pair; } \\
\mathrm{nH}\end{array}$ & $\begin{array}{c}\text { Capacitance of the } \\
\text { twisted pair; } \\
\mathrm{pF}\end{array}$ \\
\hline 1 & 2700 & 500 & 22 \\
\hline 0,5 & 1208 & 250 & 11 \\
\hline 0,25 & 534 & 125 & 5,5 \\
\hline
\end{tabular}

Finally, impedance $\mathrm{Z}_{\mathrm{LC}}$ of an equivalent $\mathrm{LC}$ circuit created by the twisted pair can be determined as follows:

$\left|\mathrm{Z}_{\mathrm{LC}}\right|=1 /(1 /(\omega L)-\omega C)$

Where $L$ - inductance, $C$ - capacitance, $\omega=2 \pi f ; f$ - frequency

The results of this calculation are shown in the graph (Fig7) for frequencies ranging from $100 \mathrm{kHz}$ to $100 \mathrm{MHz}$. This is the range where 96\% of HEMP's power is emitted [3].

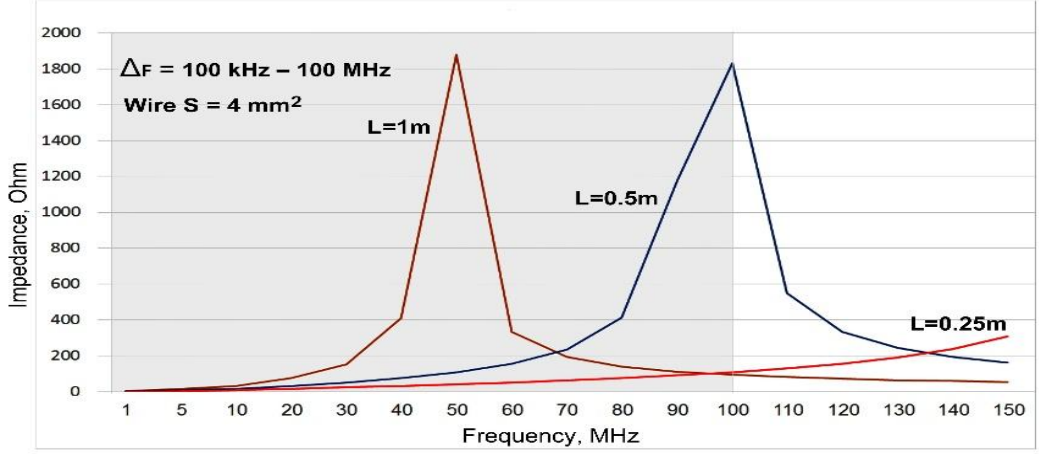

Figure7. Impedance of a twisted pair of mounting wires of different lengths as a function of frequency.

The obtained results are unexpected to some degree. The fact is that this range produces resonance which results in a significant increase of the twisted pair's impedance and thus protection of equipment becomes absolutely inefficient. Indeed, when the varistor is actuated and a current pulse of several hundreds of Amps (and even several kilo-Amps) starts flowing through it, the voltage drop in wires connecting the varistor with a protected item can reach dozens of kilovolts. Acceptable results can be achieved with a wire length of up to $25 \mathrm{~cm}$, but it is very difficult to implement this design in the existing cabinet full of equipment. Clearly, other factors will influence the frequency of this resonance, e.g. capacitance and inductance of arrangement and metal parts of the cabinet which are impossible (and not necessary) to account for as they will differ from cabinet to cabinet. However, it is necessary to account for the risk of resonance which results in significant decrease of protection efficiency. What should be done in this situation? 


\section{Suggested Solution Of THE Problem}

Surprisingly, but at a first glimpse, the suggested solution focuses on the necessity to locate the module with protecting elements as far away from the object being protected as possible. However, on the other hand, this is contradictory to the results obtained earlier. In fact, a new place for the module would be an additional DIN rail mounted close to the main input terminal, where the cores of the external cable which enter into the cabinet are set apart (Fig8)

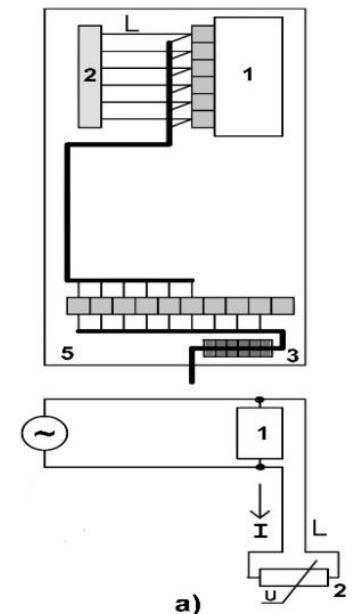

a)
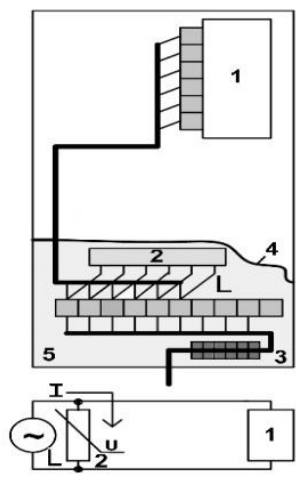

b)

Figure8. Two options for locating module 2 with varistors in the cabinet 5: a) close to equipment being protected 1; b) near input terminal, 3 - a set of ferrite rings in plastic holders with a locker placed over the input multicore control cable [1]; 4 - shielding conductive fabric, which separates "dirty" part of the cabinet from the "clean" zone with equipment.

Figure8 shows that the length $\mathrm{L}$ of the conductors through which high-amperage pulse current of varistor discharges is flowing (see option "b") can be much shorter than that of the conductors in option "a"). This layout ensures lack of resonance and acceptable level of impedance (Fig7) and thus much lower impulse overvoltage on the input terminals of equipment being protected:

$\mathrm{U}_{(1)}=\mathrm{U}_{(2)}+2 \mathrm{IZ}_{\mathrm{L}}$

Where: $\mathrm{U}_{(1)}-$ impulse overvoltage on inputs of equipment being protected;

$\mathrm{U}_{(2)}$-varistor's clamping voltage;

I - discharge current of varistor;

$\mathrm{Z}_{\mathrm{L}}$ - impedance of conductors connecting varistors with equipment being protected.

Moreover, combined action of ferrite rings [1] and varistors makes it possible to significantly suppress the amplitude of HEMP right at the input terminal. Consequently, this allows the separation of the "dirty" (from electromagnetic emissions stand point) area of the cabinet from the "clean" (with no HEMP emission) area by means of a shielding conductive fabric (4) (WU1) [1] Special chokes designed for high-amperage current (Fig9b [1]) can filter current circuits and power circuits instead of ferrite rings placed over a multi-core control cable (Fig9a).

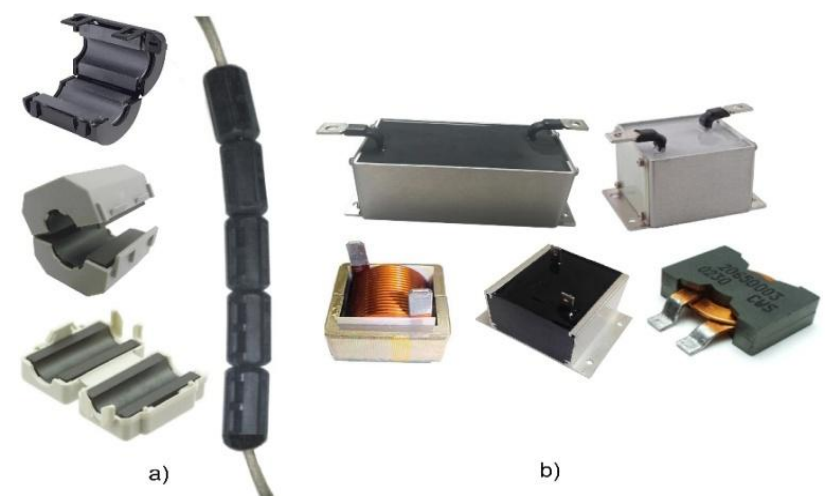

Figure9. High-frequency ferromagnetic filters utilizing ferrite rings in plastic holders with a locker (a) and special chokes $(b)$. 


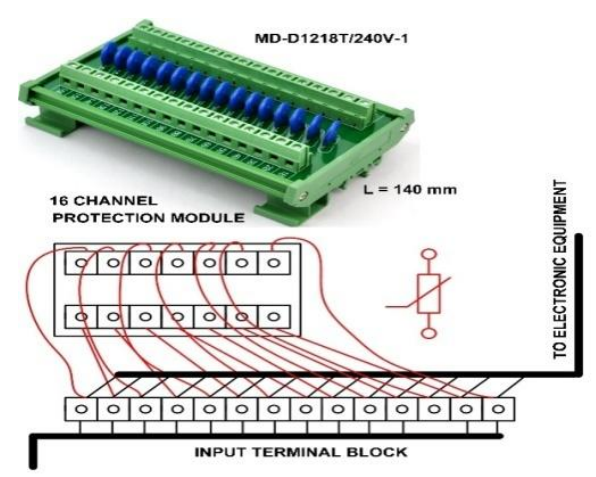

Figure10. Assembly of varistor module placed near input terminal

However, placing a varistor module near the input terminal results in too many conductors of various cross-sections in the zone of the input terminal (Fig10). At the same time, connecting the control cable's cores with a cross-section of $2.5 \mathrm{~mm}^{2}$ and conductors with a cross-section of $4 \mathrm{~mm}^{2}$ to the same terminal is not acceptable.

An alternative design of a varistor module (Fig11) can solve the problem.
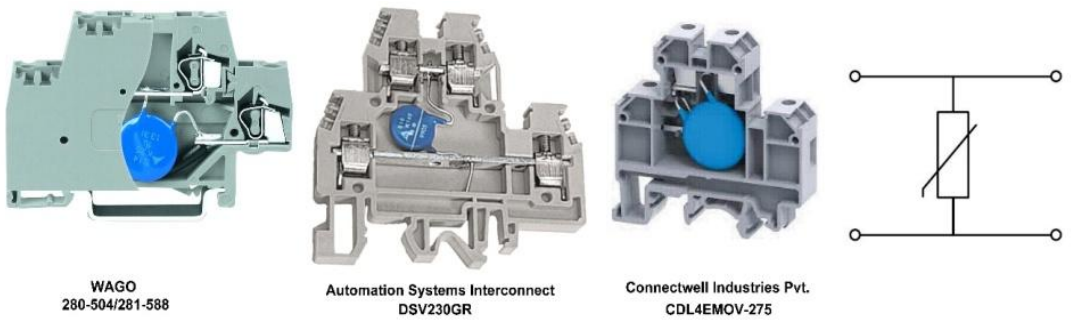

Figure11. Varistor modules utilizing individual sections of a standard terminal block designed to be mounted on a DIN rail.

The design of these modules shows that varistor outputs are connected to the input terminal between the terminal and the cores of internal control cables rather than parallel to the cores and thus establishing a so-called "pass-through" circuit with two terminals per each output of the varistor. With this approach to varistor connection, it is unnecessary to connect two cores with different crosssections into one terminal. Moreover, this makes assembly much easier and orderly (Fig12).

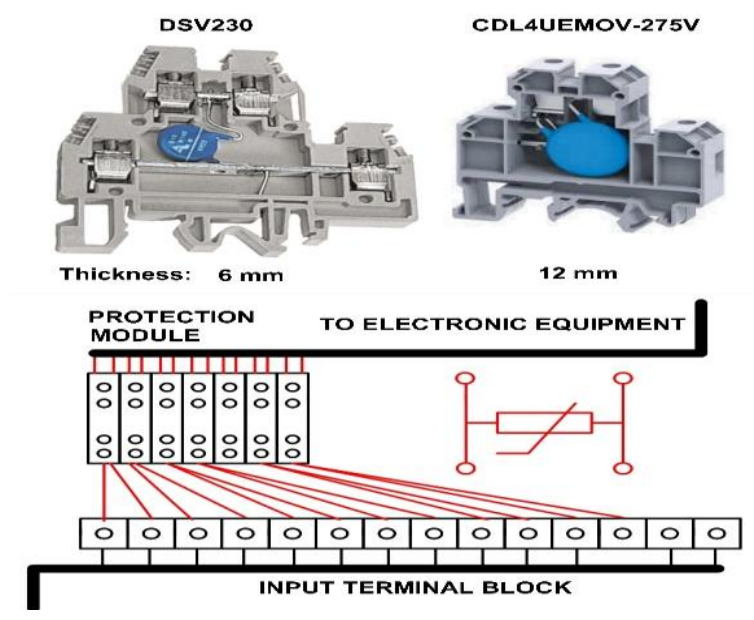

Figure12. Assembly of recommended varistor module placed near input terminal.

Unfortunately, varistor modules which meet the requirements in terms of parameters and internal connection circuit are very rare in the market, only two modules of such type were found (Fig12). The problem is that many companies manufacture such modules with relatively low-power varistors for low operating voltages and for wires with a cross-section of up to $2.5 \mathrm{~mm}^{2}$.

The used built-in varistor in DSV230 does not generate more than $2.5 \mathrm{kA}$ amplitude of discharge current and this is not sufficient to ensure HEMP protection. The varistor used in CDL4UEMOV$275 \mathrm{~V}$ is much more powerful and allows the pulse current flow with amplitude of up to $6.5 \mathrm{kA}$. 
However, this module is two times thicker compared to the DSV230 (12 $\mathrm{mm}$ vs $6 \mathrm{~mm}$ ) and this feature is very important in case there is a necessity to install many modules like this.

Considering the differences in power and thickness of the modules, it would be a good idea to use DSV230 to protect the equipment's inputs to which control cables entering the room with cabinets are connected, while CDL4UEMOV-275V can be used to protect inputs to which long cables exiting the room are connected (secondary circuits of high-voltage external current and voltage transformers and powersupply circuit).

Since varistors permit the flow of a limited number of pulses with an amplitude of thousands of amperes (afterwards their parameters deteriorate), they should be selected based on a voltage parameter in order to prevent their actuation under lightning charge or switching over voltages, i.e. their actuation voltage should be higher than what is usually used to protect electronic equipment in electric circuits. For example, while varistors with maximum continuous voltage not exceeding 275 $\mathrm{V}$ are used for nominal voltage of $230 \mathrm{VAC}$, it is advisable to use varistors with maximum continuous voltage of $320 \mathrm{~V}$ for HEMP protection. Higher clamping voltage of these varistors falls within the range of acceptable values for industrial equipment meeting the requirements of common EMC standards.

The best way out for these modules would be to use varistors suitable for HEMP protection and featuring the size of those that are used in mass-produced modules. In this case it would be easier to contact a manufacturer and order a batch of modules with necessary varistors (Table2).

Table2. Recommended types of varistors to be used in protection modules

\begin{tabular}{|c|c|c|c|c|}
\hline Type/Manufacturer & Littelfuse & Epcos & Vishay & Bourns \\
\hline Varistor's Type & V320LA20CP & S14K320E2 & VDRS14T 320xyE & MOV- 14D511K \\
\hline Diameter, mm & 14 & 14 & 14 & 14 \\
\hline Max. OperatingAC Voltage, $\mathrm{V}_{\text {RMS }}$ & 320 & 320 & 320 & 320 \\
\hline Varistor Voltage, V & 558 & 510 & 510 & 561 \\
\hline Max. Absorption Energy, J & 165 & 136 & 120 & 125 \\
\hline Max. Pulse Current $(8 / 20 \mu \mathrm{s}), \mathrm{A}$ & 6500 & 6000 & 4500 & 4500 \\
\hline Clamping Voltage, V & 850 & 840 & 842 & 845 \\
\hline
\end{tabular}

One element of a module would cost approximately 10 USD for an end user. Actually this is affordable especially if we consider the cost of equipment that these modules protect and the inability of quick substitution of equipment not protected by these devices upon HEMP impact.

\section{CONCLUSION}

1. Large numbers of varistors necessary to protect equipment and limited space in existing cabinets, stipulate the use of special modules designed to be mounted on a standard DIN rail which contain varistors and terminals to connect external mounting wires.

2. The most suitable design would be the "pass-through" modules with four terminals: two terminals for each varistor's output.

3. The market offers varistors with more suitable parameters for HEMP protection compared to those that are used in mass-produced modules. This justifies ordering modules with varistors featuring parameters described in the article.

4. The length of conductors connecting varistors with a protected object should be as short as possible, but not longer than $25 \mathrm{~cm}$. Conductors connecting each varistor with a protected object should be designed in the form of a twisted pair made of $4 \mathrm{~mm}^{2}$ (cross-section) mounting wire.

5. The most suitable location of a varistor module in the cabinet would be the space near the input terminal block.

6. HEMP protection should be supplemented by simple ferromagnetic filters, utilizing ferrite rings placed over low-current multi-core control cables, and chokes connected into current and power circuits' break.

7. Varistor modules input terminal block, ferrite rings and chokes must be densely located near the entrance point of external cables and separated from the rest of the cabinet by a shielding conductive fabric. 


\section{REFERENCES}

[1] Gurevich V. Protecting Electrical Equipment: Good Practices for Preventing High Altitude Electromagnetic Pulse Impacts, De Gruyter, Berlin, 2019.

[2] Grower F. W. Inductance Calculations: Working Formulas and Tables. - D. Van Nostrand, 1946 (reprint: Dover, 2004).

[3] IEC 61000-2-9 Electromagnetic compatibility (EMC) - Part 2: Environment - Section 9: Description of HEMP environment - Radiated disturbance, 1996.

\section{AUTHOR'S BIOGRAPHY}

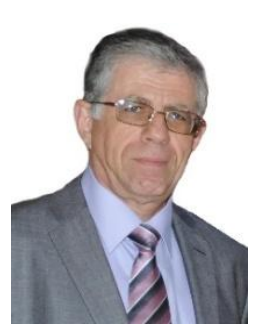

Vladimir I. Gurevich was born in Kharkov, Ukraine, in 1956. He received an M.S.E.E. degree (1978) at the Kharkov Technical University, named after P. Vasilenko, and a Ph.D. degree (1986) at Kharkov National Polytechnic University. His employment experience includes: teacher, assistant professor and associate professor at Kharkov Technical University, and chief engineer and director of Inventor, Ltd. In 1994, he arrived in Israel and works today at Israel Electric Corp. as a Senior specialist and Head of section of the Central Electric Laboratory.

He is the author of more than 200 professional papers and 15 books and holder of nearly 120 patents in the field of electrical engineering and power electronics. In 2006 he was Honorable Professor with the Kharkov Technical University.

Citation: Vladimir Gurevich, "HEMP Protection of Electronic Equipment Located in Control Cabinets", International Journal of Research Studies in Electrical and Electronics Engineering, 5(2), pp 1-8. DOI: http://dx.doi. org/10.20431/2454-9436.0502001

Copyright: (C) 2018 Authors. This is an open-access article distributed under the terms of the Creative Commons Attribution License, which permits unrestricted use, distribution, and reproduction in any medium, provided the original author and source are credited. 